\title{
Adaptive Scheduling Design for Time Slotted Channel Hopping Enabled Mobile Adhoc Network
}

\author{
Sridhara S.B ${ }^{1}$ \\ Professor, Dept. of ECE \\ Vijaya Vittala Institute of \\ Technology \\ Bengaluru, India
}

\author{
Ramesha $\mathrm{M}^{2}$ \\ Assistant Professor, Dept. of ECE \\ GITAM School of Technology, \\ GITAM (Deemed to be University) \\ Bengaluru, India
}

\author{
Veeresh Patil ${ }^{3}$ \\ Professor, Dept. of ECE \\ Amruta Institute of Engineering and \\ Management Science \\ Bengaluru, India
}

\begin{abstract}
Industrial Internet of things (IIOT) applications comprises the wearable sensor devices for human activity monitoring; these devices generate the continuous data at higher data rate and it is powered through the battery. Hence, it restricts the uses of wireless protocol such as IEEE 802.15.4 and BLE (Bluetooth Low Energy). Moreover, there are promising technologies such as TSCH (Time Slotted Channel Hoping) MAC (Medium Access Control) which can be deployed in the different environment, which are prone to interference. In this research work we focus on overcoming the issue for designing the AS (Adaptive Scheduling) for TSCH - MANET (Mobile Adhoc Network); furthermore it is very difficult to design scheduling technique considering the unpredictable nature of data source location and wireless link, this results in waste of reserve resource. Moreover proposed Adaptive Scheduling model allows the both slots i.e. shared and dedicated slots, it also allows the communicating device to active the assigned slots adaptively. Hence, our proposed AS model achieves the higher overall access fairness, minimal idle listening overhead, higher packet deliver rate; further to cope up with the higher traffic load MANET device can activate the additional slots dynamically. Moreover, the outcome of Adaptive Scheduling model shows the higher data transmission and lower energy consumption.
\end{abstract}

Keywords-6TiSCH; access fairness; energy efficiency; MANET (Mobile Ad Hoc Network); TSCH (Time Slotted Channel Hopping); scheduling

\section{INTRODUCTION}

Modern applications service of communication requires different characteristics such as scalability, adaptability, dependability and low idleness, However to develop such model it requires high maintenance and high cost. Furthermore, advancement in wireless tries to fulfill the performance guidelines through proposing new mechanism. IEEE 802.15.4 (2015 standards) is recently developed prototype and it is known as the modified version of IEEE802.15.4 (2006 standards) [1]. It acts as the empowering agent remote PAN (Personal Area Network), it possesses the characteristics of SCFR (Short Communication Frequency Range), low bandwidth and low powered [2], best example of this kind model is Sensor Network and MANET(Mobile Adhoc Network). 2015 standards of IEEE 802.15.4 develop the TSCH mode, which is very much feasible in providing the strength to fading ANF multipath interference. Furthermore rise of IPV6 over 6TiSCH [3] helps in providing the Internet Protocol organized abilities in case of low powered TSCH and thus it fills the gap between the modern technologies environments.

In 6TiSCH model, the data link layer is available on the IEEE 802.15.4-TSCH, in 802.15.4 TSCH MAC, MANET adopts the scheduling technique and it synchronizes on the frame organization. However, 802.15.4 - TSCH fails to explain modelling of scheduling method. In recent years several researchers has been developed for packet scheduling in the 6TiSCH, furthermore it is observed that scheduling is modelled through distributed or centralized manner. However In earlier method, MCS (Main Control Server) is responsible for the constructive scheduling mechanism, Moreover $n$ case of decentralized environment condition, global system information and main controller in formation is not accessible. Hence, MANET devices acknowledge the scheduling mechanism through the neighboring devices by process of schedule optimization and interchange change of data in device to device. Centralized technique [4][5] are mainly suitable for the static network where overhead is nominal, further the centralized based scheduling mechanism is suitable for the large MANET device, however the cost plays an important role. In case of decentralized technique, this is mainly applicable mainly for the huge scale system and which is dynamic in its nature. Hence it is observed that in comparison with centralized, decentralized based brings the better energy utilization.

Moreover, energy efficient results in reduction in energy overhead [6] for MANET routing [7]. IoT observes the huge application such as smart cities, smart homes and wearable devices, further the IoT application requires flexible and robust characteristics, and apart from this minimum energy, consumption is required and further support the huge operation. In this research work, the main aim is to enhance the energy efficient and data transmission of TSCH network. Moreover, TSCH is parted based on high data rate and dynamic traffic; moreover, this is characterized due to dynamic nature of WNC (Wireless Network Channel) condition. Hence, retransmission amount is random, further In case of High Bandwidth Network the packet reception capability of the MANET Device is utilized to its full potential. Nevertheless, this phenomena occurs mainly due to the various aspects, first aspect is that it is highly improbable to forecast the data rate and mobile location, second aspect is that it is very difficult to adopt and deal with dynamic nature of the wireless network, 
third it is very difficult to find the change in route. Meanwhile it is also observed that it timescale variation in these changes are in terms of milliseconds. In the above, three condition the scheduling does not fit, this means it is non-practical to design and share in reactive fashion. Hence, to avoid that static allocation is needed over the slot allocation, however this might result in reduction in completely scheduling capability and this might result in many reserve slots of TSCH left unused. Moreover, it is also observed that conservative over the allocation might cause in packet loss increment, this occurs due to lack in scheduling capacity catering the high volume traffic is difficult. Similarly, there might occur energy overhead due to excessive TSCH slot allocation. Furthermore, the assigned slot are not fully utilized by the transmitter and causes the idle listening overhead in receiver. Hence, to overcome the above-discussed problem this research work proposes the AS aka Adaptive Scheduling for the MANET (TSCH enabled). Adaptive Scheduling aka AS is designed through extending Static scheduling this further allows the receiver and transmitter to choose the time-slots based on the properties of slot utilization.

\section{The contribution of work is as follows:}

- This paper presents the novel AS aka Adaptive Scheduling model for mobile Adhoc Network that is TSCH enabled.

- Adaptive Scheduling achieves the satisfactory tradeoff between the energy and packet transmission performance.

- The main advantage of Adaptive Scheduling is that it provisions for the high traffic application and this does not have any effect on the energy consumption of MANET, furthermore this is achieved through activating the additional slots dynamically and it does not require the reorganizing of new schedule.

- The Adaptive model outperforms various state-of-art technique such as [8], [10], [11], [12], [13] and [14] in terms of access fairness, packet transmission and energy efficiency.

The paper is articulated as follows: Section I gives brief introduction of scheduling mechanism using $\mathrm{TSCH}$ for wireless and mobile adhoc network. Further, highlights research problem, issues and challenges in designing adaptive scheduling design. In Section, II the proposed adaptive scheduling model for TSCH enabled mobile adhoc network is presented. Experiment result and analysis is discussed in Section III. Lastly, the conclusion with future research direction of work is discussed.

\section{ADAPTIVE SCHEDULING MODEL FOR TSCH ENABLED MOBILE ADHOC NETWORK}

In this section, we discuss the AS aka Adaptive Scheduling for TSCH enabled MANET, further this particular research focus on designing the efficient scheduling mechanism, which achieves the satisfactory tradeoff between the maximizing packet transmission and minimizing the energy efficiency. Here at first system model is design, followed by that we design energy model for computing the energy dissipation per packet, later the tradeoff model is developed for energy minimization, last but not least this section presents the Adaptive Scheduling.

\section{A. System Model}

Let's consider the high traffic network that has high data rate and it comprises the huge amount of MANET devices that performs under the dense mobile adhoc network, however considering such environment TSCH based slotted aloha does not perform well due to collision occurrence. Meanwhile packet transmission is restricted once the slots are allotted in individual manner and it causes restriction in catering devices. Moreover let us consider the TSCH network (single Hop based), which comprises the dingle sink device and two-end device $X$ and $Y$, these end devices communicates with the bandwidth $s_{X}$ and $s_{Y}$ respectively. Further, these end devices towards the sink are describes through the DLL(Data Link Layer) packet reception rate $q_{X}$ and $q_{Y}$ respectively, meanwhile this research work considers the retransmission as the independent Bernoulli distribution Set that has the similar PRR in case of each distribution. Moreover, equation 1 computes the cumulative predictable transmission denoted as $S_{X}$ for the device $X$.

$$
S_{X}=s_{X} \sum_{o=1}^{\infty} o q_{X}\left(1-q_{X}\right)^{o-1}=\frac{s_{X}}{q_{X}}
$$

In similar fashion, for device $Y$ predictable number of transmission is computed and denoted by $S_{Y}$

$$
S_{Y}=s_{Y} \sum_{o=1}^{\infty} o q_{Y}\left(1-q_{Y}\right)^{o-1}=\frac{s_{Y}}{q_{Y}}
$$

$S_{X}$ in addition, $S_{Y}$ are known PPS (Packet per Slot)-frame, amount of slots in the particular slot frame is denoted by $O_{G}$, where $O_{T} \leq O_{G}$ indicates the slots shared within the slot frame; furthermore here each MANET device comprises $\frac{1}{2}\left(O_{G}-O_{T}\right)$ contention less slots.

Here each end devices tries to utilize the dedicated slot first i.e. Until $O_{E}$ packets for each slot frame, later it transmits the residual packet, hence excess PL (Packet Load) is formulated through the below equation.

$D_{X}=S_{X}-O_{E}$

And

$D_{Y}=S_{Y}-O_{E}$.

In case of non-availability of shared $\operatorname{slots}\left(O_{T}=0\right)$, excess packet loss is also assumed lost; else if the slots are available then it is communicated through shared medium with collision $\frac{D_{X}}{O_{T}}$ and $\frac{D_{Y}}{O_{T}}$ in respective manner. Later cumulative packet collision which is denoted by $L$ is formulated through the below equation and considered as the likelihood of end devices $X$ and $Y$ that chooses the same slots.

$L=O_{T} \frac{D_{X}}{O_{T}} \frac{D_{Y}}{O_{T}}=\frac{D_{X} D_{Y}}{O_{T}}$.

Further end-to-end PDR (Packet Delivery Rate) is computed foe end device $X$ through the below equation. 


$$
P D R_{X}=\left\{\begin{array}{lr}
1-\frac{D_{X}}{S_{X}} & O_{T}=0 \\
1-\frac{L}{S_{X}} & O_{T}>0 \text { and } D_{X} \leq O_{T} \\
1-\frac{L+D_{X}-O_{T}}{S_{X}} & O_{T}>0 \text { and } D_{X}>O_{T} .
\end{array}\right.
$$

Similarly PDR (Packet Delivery Rate) for the end device $Y$ is computed through the below equation.

$$
P D R_{Y}=\left\{\begin{array}{lr}
1-\frac{D_{Y}}{S_{Y}} & O_{T}=0 \\
1-\frac{L}{S_{Y}} & O_{T}>0 \text { and } D_{Y} \leq O_{T} \\
1-\frac{L+D_{Y}-O_{T}}{S_{Y}} & O_{T}>0 \text { and } D_{Y}>O_{T} .
\end{array}\right.
$$

Furthermore, the system performance is optimized through the shared timeslot $O_{T}$ and maximize the average PDR (Packet Delivery Rate).

\section{B. Energy Consumption Model}

In order to design the energy consumption model this particular research consider the contention free schedules, hence at first unicast transmission is assumed and here each slot time is categorized into TxRx slot, idle listening slot and sleeping slot. Furthermore, transmitter and receiver is considered to be in the sleeping state and their radio is turned off. Moreover TCH scheduled slot is considered as the unused slots, further cumulated energy dissipation is computed through the below equation (this is considered in the sleeping stage):

$E_{s l p}=O_{s l p} \cdot\left(2 . O_{s l p} \cdot W\right)$,

In the above equation, $O_{s l p}$ is electric charge in the sleeping slot process for the given voltage $W$, here $W$ indicates the MANET supply voltage. Moreover, factor 2 indicates both receiver as well as transmitter, in case of idle listening slot; for initializing the communication between the transmitter and receiver a particular slot is dedicated. However In here none pending packet is observed among the sender, Hence energy wastage is induced in case of Idle listening slot, further the cumulated $\mathrm{ED}$ (Energy Dissipation) can be computed through the below equation:

$E_{\text {idlsn }}=O_{\text {idlsn }} \cdot\left(U_{\text {slp }} \cdot W+U_{\text {idlsn }} \cdot W\right)$,

Moreover in above equation, $U_{\text {idlsn }}$ indicates induced electric charge among the receiver for idle listening slot stage; further In case of TxRx slot, for communication between the transmitter and receiver, a particular slot has been dedicated. Hence, Transmitter is switched on to perform on the transmitter and meanwhile the receiver observes and listens to the given channel for further communications. Meanwhile, pending packet queues possesses minimum one frame for performing the transmission, at last cumulated ED (energy Dissipation) for the TxRx slot can be computed through the below equation:

$E_{\text {trnsrecv }}=O_{\text {trnsrecv }} \cdot\left(U_{\text {TrnsDatRecvAck }} \cdot W+\right.$

$U_{\text {RecvDatTrnsvAck }} \cdot W$ ),

Moreover in above equation, $U_{\text {TrnsDatRecvAck }}$ indicates the induced electric charge to perform the data transmission among the transmitter and acknowledgement packet, similarly $U_{\text {RecvDatTrnsvAck }}$ depicts the induced electric charge among receiver. Nevertheless, these parameters are described as the upper limit in case of failed transmission; this occurs due to channel error or any presence of interference. Moreover, in this work, it is taken as the successful transmission and further it is incremented with $O_{\text {trnsrecv }}$. Hence, consumption of average energy per packet is formulated as below.

$\mathbb{E}=\frac{E_{\text {slp }}+E_{\text {trnsrecv }}+E_{i d l}}{s \cdot F R A M E S}$

Here $s$ indicates the packet load incoming, which is further described as the packets per frame, similarly FRAMES indicates the total number of simulated frames.

\section{Energy Minimization Tradeoff Model}

In this sub-section, we focus on the trade-off model between the reliable packet transmission and energy minimization for the TSCH enabled MANET; further, it is assumed that there are eight active slots always active for transmission. Later we minimize the active slots while reducing energy dissipation of radio. In case if the slot size is still less than eight then this might not be sufficient for the relaying the packet load, in case if slot size is still more than 8 then due to idle listening the energy gets wasted. Hence, to achieve the good trade-off we define $\mu$ is the energy per packet to perform the packet delivery efficiently [14], [15] [16].

$\mu=\frac{\mathbb{E}}{P D R^{o}}$,

In the above equation $o$ helps in regulating the tradeoff specification of given reliability over the energy dissipation, meanwhile lower value of $\mu$ indicates better scheduling of MANET (TSCH based) [17], [18], [19].

Moreover proposed Adaptive scheduling model provide assurance of access fairness among the adjacent contending devices, further in the communication slot, given MANET device can select any adjacent device which has non-empty data packet buffers as receiver. Furthermore it is observed that employing the adjacent device for the personal gain leads to the bandwidth starvation in case of low prioritized devices, mainly due to the condition where the data in adjacent queues are not transmitted before the expiration. In this work, the selection process has been carried out in the round robin manner through utilizing the available timeslots as input; further considering $O$ as the MANET device, which has the information regarding device's channel offset [20]. Moreover, the proposed algorithm provides the equal priority to the neighbor until the slot frame length and $O$ are of co-prime numbers, this provides the proposed adaptive scheduling to minimize the packet drop rate in comparison with the existing model. Hence, considering the above scenario, it is clear that proposed model assures the access fairness; later it minimizes the energy consumption of given MANET devices and similarly we achieve the better packet transmission in comparison with the existing model. Moreover, the proposed model is evaluated in the next section of this research work. 


\section{RESULT AND DISCUSSION}

In this section, the Adaptive Scheduling is evaluated and compared with the existing scheduling technique [8], further for evaluation we have used I-5 processor packed with $12 \mathrm{~GB}$ RAM; 6TiSCH simulator is used and it is written using the python programming language through the associate of 6TiSCH WG [9] which is open source. Moreover, the existing model [8] and proposed model are incorporated in given $6 \mathrm{TiSCH}$ simulator; furthermore, the simulation parameter is given in Table I and parameter consideration is in accordance with the industrial environmental condition where the heavy traffic load is occurred [10]. Evaluation is carried out in terms of packet routing performance and energy overhead through comparing existing and propose AS model.

\section{A. Energy Consumption Perforamcne Evaluation}

This particular section gives the comparison analysis of proposed model over the existing model in terms of energy consumption; the evaluation is performed by considering the various transformation rate and packets. Moreover, Fig. 1. depicts the comparison of existing and proposed model by varying packet number; in here, the packet variation is from 3600 to 7200 . Furthermore, through the Fig. 1, it is observed that energy consumption of proposed Adaptive Scheduling for $3600,4800,6000,7200$ packets is $15.22 \%$, 2283\%, $27.99 \%$ and $32.78 \%$ lesser than the existing model. Meanwhile the average energy consumption is reduced by $24.77 \%$ when compared to the existing model.

\section{B. Throughput and Packet Delivery Rate Perforamcne Evaluation}

In this section, the comparative analysis of existing and proposed model based on packet routing performance such as throughput is evaluated, the evaluation is carried out varying the transmission rate(in Mbps). Fig. 2 shows the throughput comparison of existing and proposed model, here we observe that performance of conventional static algorithm is satisfactory till the TR (Transmission rate) of 6 MBPs. In case of higher transmission rate the existing model underperforms whereas proposed Adaptive Scheduling performs satisfactory until $20 \mathrm{Mbps}$, furthermore proposed model achieves the $47.83 \%$ enhancement in comparison with the existing model.

\section{Access Fairness Performance Perforamcne Evaluation}

In this sub section of performance evaluation the comparative analysis of existing and proposed model is carried out based in the access fairness; further the evaluation is carried out by varying the number of packets. Fig. 2 shows the packet drop rate comparison of existing and proposed model; it is observed that packet drop rate of proposed model is very low when compared to the existing model. Moreover, the packet drop rate of proposed model is reduced by $99.7 \%$ over the existing model, this shows the significant access fairness of proposed Adaptive scheduling model.

Energy consumption for varied packets

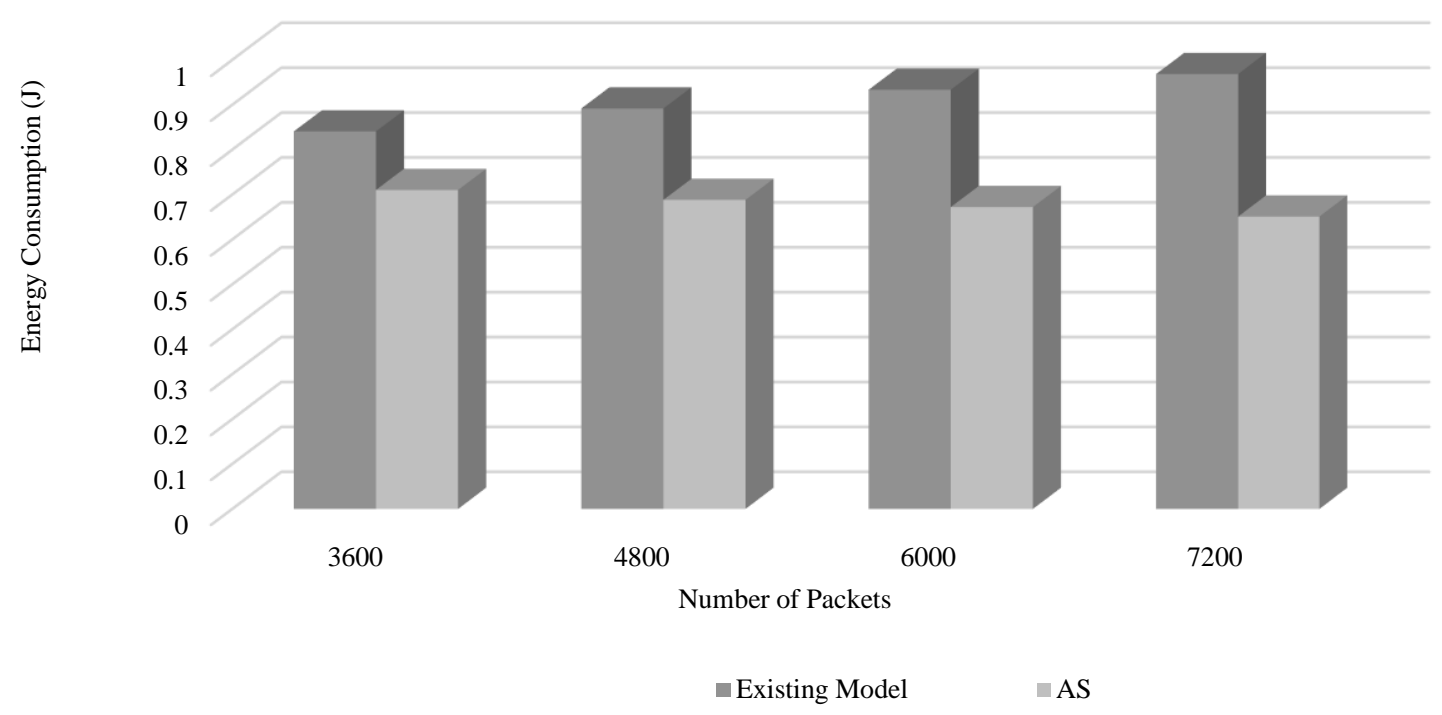

Fig 1. Energy Consumption Performance Evaluation Considering Varied Packets. 
Throughput achieved for varied transmission rate

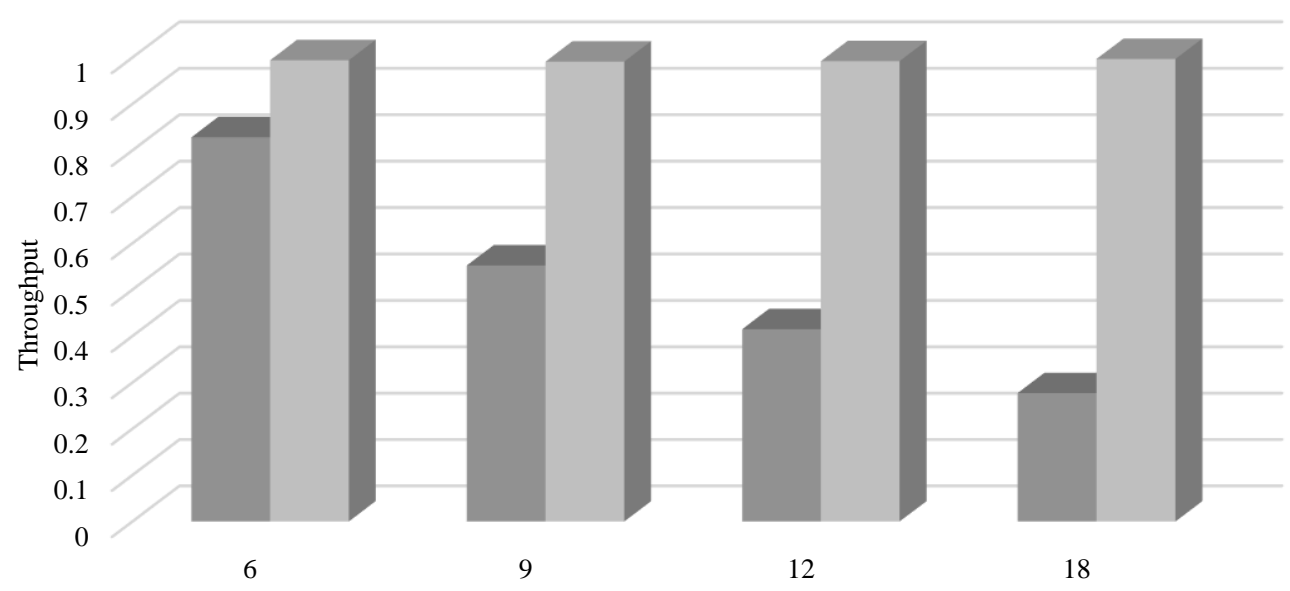

Transmission Rate in Mbps

Existing Model $\quad$ AS Model

Fig 2. Throughput Performance Achieved Considering Varied Transmission Rates.

\section{Comparision with Existing Model}

In this section we discuss the evaluation of different model when compared to our exiting model; in [11] author carried out the huge survey of existing model by addressing the performance issues for the real time application. Moreover, [8] proposed a scheduling technique, which considers the decentralized network, and this technique achieves the $44.5 \%$ improvisation in throughput, however the energy consumption and packet routing performance is ignored. Similarly, [10] and [12] performed the evaluation and achieves the energy consumption minimization of $27.08 \%$ and $25.6 \%$, respectively; whereas [13] achieved the energy overhead reduction of $22.38 \%$ and packet dropt rate of $25 \%$ when compared to the existing technique. Moreover, we compare our model with [8] since they considered the decentralized network; it is observed that AS technique achieves the satisfactory trade-off between energy overhead and routing performance. Hence from discussion it is clear that our model outperforms the various state-of-art technique [8], [10], [11], [12], [13], and [14] considering the carious parameter such as access fairness, packet delivery rate and energy overhead.

\section{CONCLUSION}

In this research work, we consider an adaptive scheduling model for TSCH enabled MANET; through the extensive analysis, it is observed that static scheduling technique possesses the higher energy overhead; this is due to the heavy resource allocation. Moreover, it is quite difficult to develop efficient scheduling technique since the data source location and nature of wireless link are unpredictable. Hence, to achieve the good tradeoff a model named Adaptive Scheduling is proposed which allow both slots i.e. shared slots as well as dedicated slots; further, it also allows the communicating device to activate their assigned slots and this results in access fairness. Moreover to cope up with the high load traffic, MANET device can easily activate assigned slots; further the comparative analysis is carried to evaluate the proposed AS model over the existing one. Proposed AS model achieves the energy overhead minimization of $24.77 \%$ compared to the existing model; furthermore considering the throughput performance AS achieves $47.83 \%$ better and packet drop rate by $99.7 \%$ better than the existing model. Hence proposed Adaptive Scheduling technique achieves the higher access fairness than any of the state-of-art technique, further in future work we would be focusing on evaluating considering different performance parameter and under multi-hop TSCH network.

\section{REFERENCES}

[1] IEEE Standard for Low-Rate Wireless Networks, IEEE Standard 802.15.4-2015, pp. 1-709, 2016.

[2] "IEEE Standard for Local and metropolitan area networks--Part 15.4: Low-Rate Wireless Personal Area Networks (LR-WPANs)," IEEE Std 802.15.4-2011 (Revision of IEEE Std 802.15.4-2006), pp. 1-314, 2011.

[3] D. Dujovne, T. Watteyne, X. Vilajosana, and P. Thubert, "6TiSCH: Deterministic IP-enabled industrial Internet (of Things)," IEEE Commun. Mag., vol. 52, no. 12, pp. 36-41, Dec. 2014.

[4] Z. Shelby, K. Hartke, and C. Bormann, The Constrained Application Protocol (CoAP), IETF RFC 7252, 2014.

[5] I. Juc, O. Alphand, R. Guizzetti, M. Favre and A. Duda, "Energy consumption and performance of IEEE 802.15.4e TSCH and DSME," 2016 IEEE Wireless Communications and Networking Conference, Doha, pp. 1-7, 2016. 
[6] S. Bandyopadhyay and E. J. Coyle, "Minimizing communication costs in hierarchically-clustered networks of wireless sensors," Elsvier Journal of Computer Networks, vol. 44, no. 1, pp. 1-16, 2004.

[7] L. Karim and N. Nasser, "Energy efficient and fault tolerant routing protocol for mobile sensor network," in IEEE International Conference on Communications (ICC), Japan, pp. 1-5, 2011.

[8] Municio, Esteban \& Latré, Steven "Decentralized broadcast-based scheduling for dense multi-hop TSCH networks", Proceedings of the Workshop on Mobility in the Evolving Internet Architecture, Pages 1924, 2016.

[9] T. Watteyne, K. Muraoka, N. Accettura, and X. Vilajosana. The 6tisch simulator. https://bitbucket.org/6tisch/simulator/src, 2015.

[10] Kralevska, Katina \& Vergados, Dimitrios \& Jiang, Yuming \& Michalas, Angelos. (2017). A Load Balancing Algorithm for Resource Allocation in IEEE 802.15.4e Networks, 2017.

[11] Rodrigo Teles Hermeto, Antoine Gallais, Fabrice Theoleyre "Scheduling for IEEE802.15.4-TSCH and slow channel hopping MAC in low power industrial wireless networks" Journal Computer Communications archive Volume 114 Issue C, Pages 84-105, 2017.

[12] Thang Phan Duy, Thanh Dinh, and Younghan Kim "A rapid joining scheme based on fuzzy logic for highly dynamic IEEE 802.15.4e timeslotted channel hopping networks", International Journal of Distributed Sensor Networks, https://doi.org/10.1177/1550147716659424, 2016.

[13] X. Fafoutis, A. Elsts, G. Oikonomou, R. Piechocki and I. Craddock, "Adaptive static scheduling in IEEE 802.15.4 TSCH networks," 2018 IEEE 4th World Forum on Internet of Things (WF-IoT), Singapore, pp. 263-268, 2018.
[14] A. Elsts, X. Fafoutis, J. Pope, G. Oikonomou, R. Piechocki and I. Craddock, "Scheduling High-Rate Unpredictable Traffic in IEEE 802.15.4 TSCH Networks," 2017 13th International Conference on Distributed Computing in Sensor Systems (DCOSS), Ottawa, ON, 2017, pp. 3-10, 2017.

[15] Hermeto, Rodrigo \& Gallais, Antoine \& Theoleyre, Fabrice. (2017). Scheduling for IEEE802.15.4-TSCH and slow channel hopping MAC in low power industrial wireless networks: A survey.ComputerCommunications.114.10.1016/j.comcom.2017.

[16] Du, Peng \& Roussos, George. (2012). Adaptive time slotted channel hopping for wireless sensor networks. 10.1109/CEEC.2012.6375374.

[17] M. Ojo, S. Giordano, An efficient centralized scheduling algorithm in ieee 802.15.4e tsch networks, in: Conference on Standards for Communications and Networking (CSCN), IEEE, 2016.

[18] Hammoudi, Sarra \& Harous, S. \& Aliouat, Zibouda \& Louail, Lemia. (2018). Time slotted channel hopping with collision avoidance. International Journal of Ad Hoc and Ubiquitous Computing. 29. 85. 10.1504/IJAHUC.2018.094400.

[19] Diab, Rana \& Chalhoub, Gérard \& Misson, Michel. (2013). Overview on Multi-Channel Communications in Wireless Sensor Networks. Network Protocols and Algorithms. 5. 112. 10.5296/npa.v5i3.3811.

[20] Wu, Yafeng \& Stankovic, John \& He, Tian \& Lin, Shan. (2008). Realistic and Efficient Multi-Channel Communications in Wireless Sensor Networks. Proceedings - IEEE INFOCOM. 12. 1193 - 1201. 10.1109/INFOCOM.2008.175. 